\title{
Convergence in Mammalian Nucleus of Solitary Tract During Development and Functional Differentiation of Salt Taste Circuits
}

\author{
Mark B. Vogt ${ }^{1, a}$ and Charlotte M. Mistretta ${ }^{1,2}$ \\ 'Department of Biologic and Materials Sciences, School of Dentistry, ${ }^{2}$ Center for Human Growth and Development and \\ Center for Nursing Research, University of Michigan, Ann Arbor, Michigan 48109-1078
}

To determine the type and extent of neural rearrangements that are made during functional differentiation of circuits for salt taste processing, we determined receptive field size and salt response characteristics of second-order taste cells in 3 age groups of sheep. Neurophysiological recordings were made from single cells in the nucleus of the solitary tract (NST) in fetal, perinatal, and postnatal sheep. Responses to $\mathrm{NH}_{4} \mathrm{Cl}, \mathrm{NaCl}$, and $\mathrm{KCl}$ were measured, and location and number of fungiform papillae in the receptive field were determined by stimulating individual papillae with anodal electrical current. The data are compared with previous, parallel measures from chorda tympani nerve afferent taste fibers to permit conclusions about convergence or divergence onto second-order cells.

Receptive field size of second-order taste neurons increases during development, in contrast to the decrease in field size observed previously for chorda tympani nerve fibers during the same period. Furthermore, receptive fields of second-order cells are significantly larger than those of first-order fibers at perinatal and lamb ages, but not fetal. Thus, there is convergence of first-order taste afferents onto brain-stem neurons, and the convergence increases remarkably between fetal and perinatal periods. Associated with the increase in convergence are increased salt response frequencies relative to afferent fibers for $\mathrm{NaCl}$ in perinatal animals and lambs, and for $\mathrm{KCl}$ in lambs. The increase in frequencies occurs before NST neurons are functionally mature, as indicated by the rapid response adaptation of many cells in young animals.

Convergence in NST during development apparently functions to maximize gain for processing neural responses to $\mathrm{NaCl}$. In the periphery, response frequencies to $\mathrm{NaCl}$ are very low in fetuses, and increase progressively during development. In the NST, $\mathrm{NaCl}$ response frequencies are high even in fetuses, and remain high. The process of convergence onto second-order cells is accomplished with maintenance of order in afferent projections because receptive fields of NST neurons are composed of fungiform papillae that are clustered together, not dispersed over the tongue.

Received Feb. 2, 1990; revised Apr. 13, 1990; accepted May 10, 1990.

This work was supported by National Science Foundation Grant BNS-8311497 and National Institutes of Health Grant DC00456 to C.M.M.

Correspondence should be addressed to Dr. Charlotte M. Mistretta, Biologic and Materials Sciences, Room 6226, School of Dentistry, University of Michigan, Ann Arbor, MI 48109-1078.

a Current address: Department of Psychology, University of Virginia, Charlottesville, VA 22903.

Copyright (C) 1990 Society for Neuroscience $0270-6474 / 90 / 093148-10 \$ 03.00 / 0$
Our quantification of taste receptive field size at 2 neural levels provides strong evidence for increasing convergence in the NST during development. Altering patterns of afferent neural input and geometry of second-order neurons may have a role in establishing convergence. The convergence has an apparently special function: to increase gain for $\mathrm{NaCl}$ taste sensation. Therefore, neural rearrangements during differentiation of salt taste pathways result in specific functional outcomes.

The receptive field of any neuron in a sensory pathway is composed of all the receptors that can influence its neural activity (Shepherd, 1983). Therefore, receptive field organization is an essential component of sensory circuits, and knowledge of receptive field development is important in understanding functional differentiation of sensory pathways.

To understand better the development and maturation of neural circuits for salt taste processing, we have made measures of receptive field size and salt response characteristics of secondorder taste cells in 3 age groups of sheep. These data can be compared with parallel measures of receptive fields and salt responses from first-order taste afferents. Receptive field size at various neural levels is determined during development through divergence and convergence of afferent input. For example, a primary sensory neuron may receive converging input from multiple receptors and then diverge to contact several secondorder cells; or several sensory neurons may converge on a single second-order cell. Thus, results from study of chorda tympani fibers and second-order taste cells provide an indication of convergence and divergence at 2 neural levels during development of the taste system.

In previous studies, the number of fungiform papillae and associated taste buds innervated by a single chorda tympani nerve fiber was determined in fetal, perinatal, and postnatal sheep (Mistretta et al., 1988; Nagai et al., 1988). We found that receptive fields of first-order taste afferents decreased in size during development; that is, a single fiber innervated fewer fungiform papillae in postnatal lambs than in younger age groups. The decrease in field size of taste fibers was accompanied by an increase in proportion of fibers that responded with highest frequency to $\mathrm{NaCl}$, compared to other salts, $\mathrm{NH}_{4} \mathrm{Cl}$ or $\mathrm{KCl}$. Furthermore, there was a correlation between receptive field size of first-order neurons and salt response characteristics. Fibers with smaller receptive fields (fewer fungiform papillae) responded with higher frequency to $\mathrm{NaCl}$; fibers with larger fields responded with higher frequency to $\mathrm{NH}_{4} \mathrm{Cl}$. These data led to the conclusion that there is an acquisition of small, highly $\mathrm{NaCl}-$ 
responsive, receptive fields during development of the peripheral taste system in sheep.

We now have parallel information on receptive field size and salt responses of second-order taste neurons in the nucleus of the solitary tract (NST) in the same age groups of sheep. Recordings were made from single NST neurons and responses to stimulation of the tongue with $\mathrm{NaCl}, \mathrm{NH}_{4} \mathrm{Cl}$, and $\mathrm{KCl}$ were measured in fetal, perinatal, and postnatal sheep. Using electrical stimulation of single fungiform papillae, the number of papillae in the receptive field of each cell was also determined.

In general, we have found that receptive fields of second-order NST neurons are larger than chorda tympani receptive fields. Furthermore, NST fields increase in size during development whereas chorda tympani fields decrease in size during the same periud. Thus, there is convergence of first-order taste afferents on to brain-stem neurons and the extent of convergence increases during development. Associated with the increase in convergence is an increase in response frequency to salt stimuli and changes in adaptation characteristics of the neurons. It appears that the extensive developmental convergence and resulting increases in salt response frequencies eradicate the correlation between receptive field size and salt taste response that was observed in the periphery. However, this convergence might be essential to maximize gain in the developing taste system and thereby ensure transmission of relatively weak peripheral input about $\mathrm{NaCl}$ taste stimuli to the CNS.

\section{Materials and Methods}

\section{Animals and surgical preparation}

Complete receptive field data were collected from Suffolk sheep in 3 age groups: 12 fetuses aged 126-136 d of gestation $($ term $=147 \mathrm{~d}), 8$ perinatal sheep aged $145 \mathrm{~d}$ of gestation to $9 \mathrm{~d}$ postnatal, and 18 lambs aged $29-58$ d postnatal. Throughout this report, animals in these age groups will be referred to as fetal, perinatal, and lamb, respectively. Lambs or pregnant ewes were anesthetized with an intravenous injection of sodium pentobarbital ( $30 \mathrm{mg} / \mathrm{kg}$ for ewes, $20 \mathrm{mg} / \mathrm{kg}$ for lambs); subsequent anesthetic was administered via an indwelling jugular catheter. Ewes or lambs were tracheotomized and received supplemental $\mathrm{O}_{2}$. Fetuses were delivered onto a table next to the ewe and were wrapped in disposable cotton pads and a water circulating heating pad (Bradley and Mistretta, 1973). Umbilical and placental circulation remained intact so that the fetus was anesthetized and maintained via the ewe. Rectal temperature and level of anesthesia were monitored continuously.

To provide access to the anterior tongue, the left side of the mouth was dissected back to the ramus of the mandible and the upper and lower lips were retracted with sutures. The animal's head was flexed down at a right angle to the body and mounted in a stereotaxic apparatus with blunt earbars. A portion of the posterior skull was removed, and the cerebellum overlying the medulla was aspirated to expose the floor of the fourth ventricle. The exposed brain stem was covered with mineral oil.

\section{Anesthesia}

As described above, we used pentobarbital anesthesia throughout our experiments and therefore the possibility of anesthetic effects must be considered. However, the consistency in results from a large number of studies employing different anesthetics in a variety of species (e.g., Doetsch and Erickson, 1970; Smith et al., 1983; Travers et al., 1986) suggests that anesthetic effects are negligible in studies of gustatory NST. When anesthetic types, including sodium pentobarbital, are compared, investigators report similar chemical response frequencies (Smith et al., 1983). In addition, investigators routinely report low spontaneous activity rates for taste neurons even in experiments with alert macaque monkeys (Scott et al., 1986) and with in vitro brain-stem preparations (Bradley and Sweazey, 1990). Anesthetized preparations are also used to study chemoreceptor and respiratory cells in more caudal NST (Madden and Remmers, 1982; Donoghue et al., 1985; Mifflin and Felder,
1988; Lawson et al., 1989), and in brain-stem areas other than the solitary nucleus, including trigeminal nuclei (e.g., Jacquin et al., 1989). Furthermore, in developmental studies of gustatory NST (Bradley and Mistretta, 1980; Hill et al., 1983) and other second-order sensory cells (e.g., Ferrington and Rowe, 1982; Fitzgerald, 1985) anesthetized preparations are used. In early development, neurons generally exhibit immature response characteristics including low response frequencies, rapidly adapting responses, and longer response latencies. The immature neurophysiological response characteristics that we report here are typical of those in other systems and we have no reason to assume that they are attributed to anesthetic effects.

\section{Receptive field neurophysiology}

With obex as the zero reference point, extracellular recordings from single taste neurons in the rostral NST were made with tungsten microelectrodes $(0.4-1.8 \mathrm{M} \Omega)$. Coordinates were based on previous studies of taste cells in NST of fetal and postnatal sheep (Bradley and Mistretta, 1980). Neural activity was monitored with a preamplifier, oscilloscope, and audio amplifier and recorded on tape with voice cues for subsequent analysis.

Single neuron recordings were identified during experiments by examining the superimposition of successive action potentials within a spike train using a storage oscilloscope and delay circuit. Consistency of waveform and amplitude were monitored. When neural data were digitized for subsequent data analysis, waveform was again reviewed. Second-order neurons were identified during recording sessions by their biphasic action potentials and distinctive response characteristics, especially receptive field size and $\mathrm{NaCl}$ responsiveness. In subsequent data analysis the very different neural response properties of the secondorder cells, compared to chorda tympani afferent fibers, were confirmed, as demonstrated in Results.

Taste neurons with lingual receptive fields were identified by responses to chemical stimuli flowed over the tongue. Cells that also responded to tactile stimulation of the tongue with a glass rod were not studied. Because NST gustatory cells usually have low or no spontaneous activity, we searched for taste neurons by stimulating the tongue alternately with $\mathrm{NaCl}$ and $\mathrm{NH}_{4} \mathrm{Cl}$ as the electrode was driven through the medulla. All chemical stimuli were followed by water rinses, until the cell returned to baseline activity levels. With adequate rinses the taste system responds reproducibly to repeated chemical stimulation for periods of at least 10 hours (Kitada et al., 1984).

When responses from a single neuron were identified, the approximate location of the receptive field was determined with localized chemical and electrical stimulation of various tongue regions. Then a standard chemical and electrical stimulation sequence was initiated. Chemical stimuli included three 0.5 m salt solutions, reagent grade $\mathrm{NH}_{4} \mathrm{Cl}, \mathrm{NaCl}$, and $\mathrm{KCl}$ dissolved in distilled water. Previous studies indicated that these salts elicit robust taste responses and that the responses alter substantially during development (Bradley and Mistretta, 1980; Mistretta and Bradley, 1983; Nagai et al., 1988). Because the purpose of the present study is to understand better the developing neural circuitry for salt taste processing and because it is difficult to hold neurons for sufficient time to both record chemical responses and map the receptive field, we limited ourselves to these salt stimuli. For each stimulation, $10 \mathrm{ml}$ of solution was applied to the tongue with a glass syringe and, after about $10 \mathrm{sec}$, the tongue was rinsed 2 or 3 times with $10 \mathrm{ml}$ of distilled water. About $30 \mathrm{sec}$ separated chemical stimulations.

After chemical responses were recorded, a dissecting microscope was used to view and map the receptive field by systematically stimulating and recording responses from individual fungiform papillae in the region of the field and its surround (Fig. 1). A monopolar, platinum stimulating electrode was connected to a Grass stimulator, and an indifferent electrode was placed under the tongue. The stimulating electrode was small enough (250- $\mu \mathrm{m}$ diameter) to stimulate individual papillae, which in fetal sheep at $130 \mathrm{~d}$ of gestation are $250-350 \mu \mathrm{m}$ in diameter and 400 $1000 \mu \mathrm{m}$ apart (Mistretta et al., 1988). In older animals, papillae are larger.

Papillae were stimulated with $10 \mu \mathrm{A}$ anodal current for about $1 \mathrm{sec}$ with about 5 -sec separations. The stimulating intensity was chosen to maximize the likelihood of eliciting a response from all papillae in the receptive field, based on study of receptive fields of peripheral fibers (Nagai et al., 1988), preliminary experiments with NST neurons, and determination of response/intensity functions for individual papillae.

Response/intensity functions were determined for 13 individual papillae in receptive fields of 3 NST neurons from perinatal animals; these 

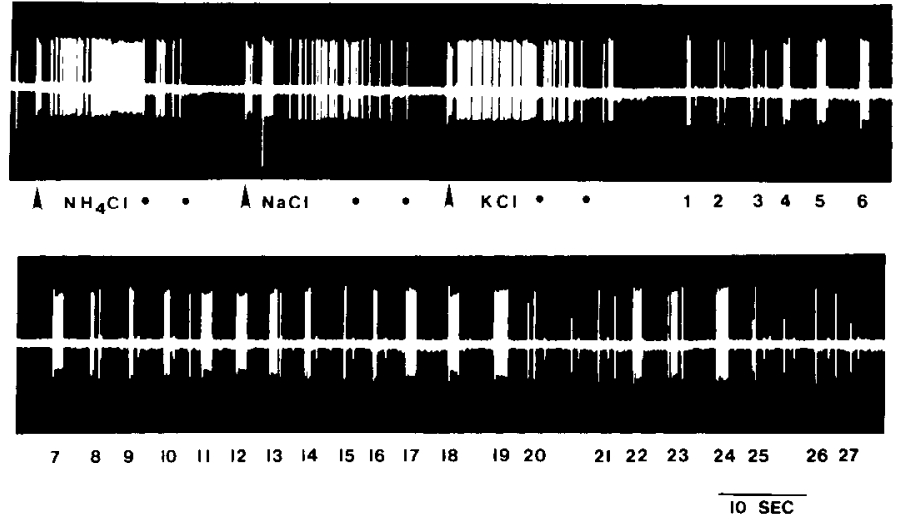

Figure 1. Chemical responses and receptive field size determination for an NST neuron in a fetus at about $130 \mathrm{~d}$ of gestation. Top, responses to $0.5 \mathrm{M} \mathrm{NH}_{4} \mathrm{Cl}, \mathrm{NaCl}$ and $\mathrm{KCl}$ are presented first. Chemicals were applied to the tongue for the period initiated at the arrowhead and concluded with dots (water rinses). Bottom, responses to electrical stimulation of individual fungiform papillae; numhers under the data represent each papilla. The receptive field contained 22 papillae. Electrical stimulation of some papillae did not elicit a neural discharge (e.g., papillae $20,21,26,27$, where only the on and offset of the electrical stimulus are seen); for others a high-frequency response was elicited (e.g., papillae 4-7).

were compared with functions for 76 individual fungiform papillae in receptive fields of 6 chorda tympani nerve fibers from perinatal animals (Fig. 2). The average functions for papillae in fields of peripheral and central neurons were similar in shape and demonstrated that $5 \mu \mathrm{A}$ elicited a response frequency at about $2 / 3$ maximum, and $10 \mu \mathrm{A}$ elicited a near-maximal response.

However, in fetal animals, response/intensity functions for 27 papillae in receptive fields of 3 NST neurons indicated that $5 \mu \mathrm{A}$ elicited a less than $1 / 2$-maximal response (Fig. 2). It seemed that peripheral input from fetal afferent fibers might not be sufficiently intense to cross presumably immature synapses onto NST cells. Therefore, based on these collective results, we decided to use $10 \mu \mathrm{A}$ stimulating intensity as a standard. In fact, receptive field sizes for 13 NST neurons mapped with both 5 and $10 \mu \mathrm{A}$ stimulating intensities were not significantly different $[t(12)=$ $1.42, p>0.10]$. Nor were field sizes different for 8 neurons when maps were determined with 10 and $20 \mu \mathrm{A}$ stimulus intensities $[t(7)=1.11$, $p>0.10]$. Therefore, our use of $10 \mu \mathrm{A}$, as opposed to a lower or higher stimulus intensity, should not have influenced the receptive field size measure.

Measures of receptive field size obtained with electrical stimulation of single papillae were reliable, as indicated by the correlation between sizes for 26 receptive fields that were mapped twice in succession $[r(24)$ $=0.98, p<0.0011$. For additional discussion of the reliability and validity of this method, see Nagai et al. (1988). Electrical stimulation at the current intensities we used stimulates taste bud cells but not chorda tympani nerve fiber endings (Herness, 1985; Frank et al., 1986). Furthermore, other sensory endings of the lingual trigeminal nerve, which innervates filiform and fungiform papillae but not taste buds, are not stimulated at such low intensities but require anodal current delivery at least 1 order of magnitude greater than we employed (Herness, 1985; Frank et al., 1986).

After recording chemical responses and mapping a receptive field, an electrolytic lesion was made at or below the recording site $(10 \mu \mathrm{A}$ direct current for $10 \mathrm{sec}$ ). At the end of the experiment the brain was removed and stored in a $30 \%$ sucrose, $10 \%$ buffered formalin solution for later sectioning, staining with cresyl violet, and histological verification of the recording site. The ewe or postnatal lamb received a lethal dose of anesthetic at the end of all procedures.

\section{Data analysis}

Recorded neural impulses were converted to standard pulses using a window discriminator. A computer was used to convert intervals between pulses to frequencies and to count the number of impulses during prestimulus and stimulus periods. All chemical and spontaneous im-

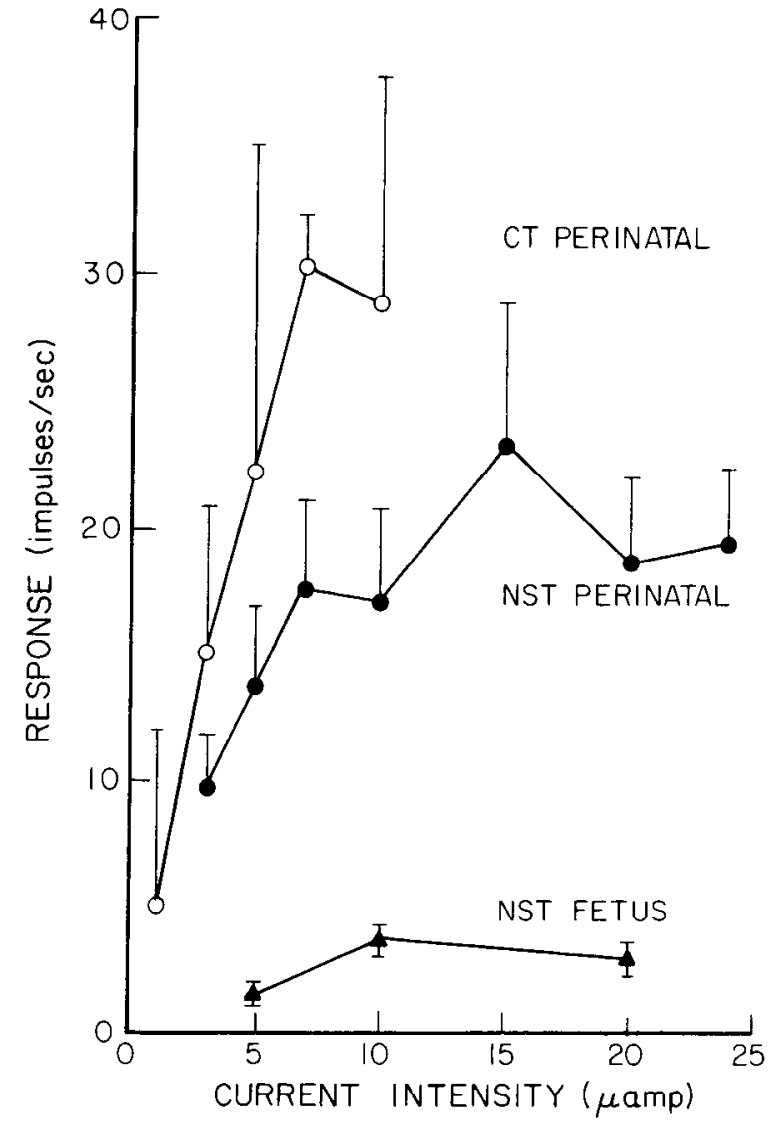

Figure 2. Response frequency as a function of current intensity used to stimulate individual fungiform papillae in receptive fields of fetal and perinatal NST neurons (filled symbols), and perinatal chorda tympani (CT) nerve fibers (open circles). Data points are means and SDs for 27 papillae in fields of 3 fetal neurons, for 13 papillae in fields of 3 perinatal neurons, and for 76 papillae in fields of 6 perinatal chorda tympani nerve fibers.

pulse frequencies are reported as impulses/sec. Chemical responses were derived from an average of response frequencies during the 5 -sec period after stimulus onset. Mean spontaneous rate was based on the 5 -sec periods that preceded each of the 3 salt stimulations.

To determine receptive field size, neural response frequency to electrical stimulation of single papillae was measured during the 1-sec period after stimulus onset. Responsive papillae were defined as those that responded to electrical stimulation with a frequency that exceeded the mean plus 2 standard deviations (SD) of spontaneous impulse frequency (for receptive field determination, spontaneous activity was measured during five 1 -sec periods that preceded electrical stimulation of the first papilla in the sequence). Receptive field size then was defined as the number of responsive papillae for each neuron. In application, the precise response criterion was unimportant because single papilla stimulation usually elicited either a marked increase in impulse frequency, or no change from the spontaneous rate.

Once a field was mapped, the location was noted on a standard tongue diagram. If the neural discharge and signal-to-noise ratio remained stable, the entire sequence of chemical stimulation and receptive field mapping was repeated, and mean values for chemical responses and receptive field size were used.

Statistical comparisons across age groups were made with analysis of variance and post-comparisons between individual groups were made using the Bonferroni procedure, with alpha divided by the number of comparisons possible within each level of the independent variable(s). The level for significance was set at $p<0.10$.

\section{Results}

Complete recording sequences, including at least one chemical stimulation series and one receptive field map, were obtained 


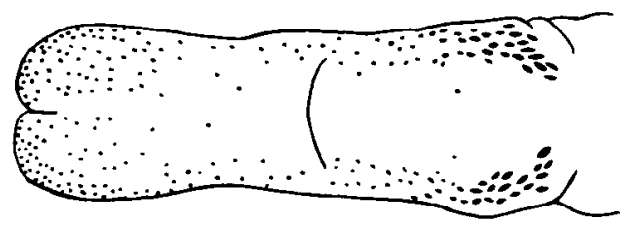
DISTRIBUTION OF PAPILLAE

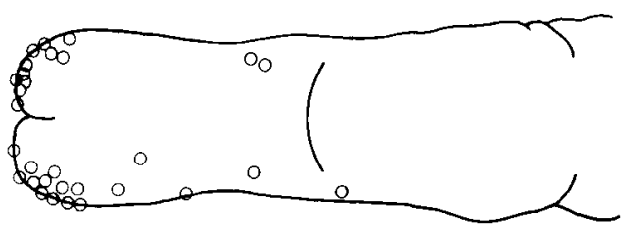

LAMB

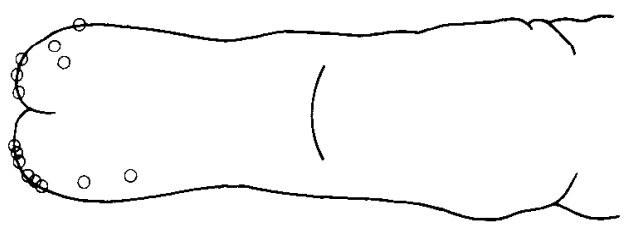

PERINATAL

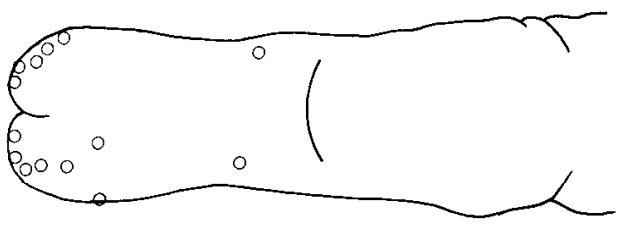

FETAL

Figure 3. Distribution of fungiform papillae on the sheep tongue (top) and locations of receptive fields of NST neurons in each age group. In the top diagram, filled ovals on the posterior tongue represent circumvallate papillae. Receptive field locations are drawn on both sides of the tongue for ease in illustration, but all fields were ipsilateral to NST cells. Similarly, for illustration purposes each receptive field is indicated as a circle, whereas in fact the fields varied widely in size (Fig. 4) and shape.

from 58 neurons: 14 in fetal, 14 in perinatal, and 30 in larnb groups. Examination of histological material to locate electrode tracks and lesions indicated that recording sites were generally in the rostral subdivision of gustatory NST, anterior to, or at the level of incoming glossopharyngeal nerve fibers. Average coordinates (and SD) rostral to obex and lateral to midline, determined at the time of recording, were fetal, $5.1(0.2) \mathrm{mm}$ rostral, $3.6(0.2) \mathrm{mm}$ lateral; perinatal, $5.8(0.7)$ rostral, $3.8(0.3)$ lateral; and lamb, $6.4(0.7)$ rostral, $4.5(0.4)$ lateral.

\section{Receptive field location and size}

All of the neurons studied had a single receptive field on the ipsilateral tongue (Fig. 3). Most receptive fields (51 of 58) were located on or near the anterior quadrant of the tongue, the region of highest density of fungiform papillae. $\Lambda$ few fields ( 2 fetal, 5 lamb) were mapped on the dorsal mid-tongue region. Some neurons had extremely posterior tongue fields, at the level of the circumvallate papillae, but these cells and receptive fields were not studied.

In general, the papillae in a receptive field were adjacent to each other and no non-responsive papillae were interspersed among responsive papillae. In addition, when recordings were made from more than 1 neuron in an animal, there could be
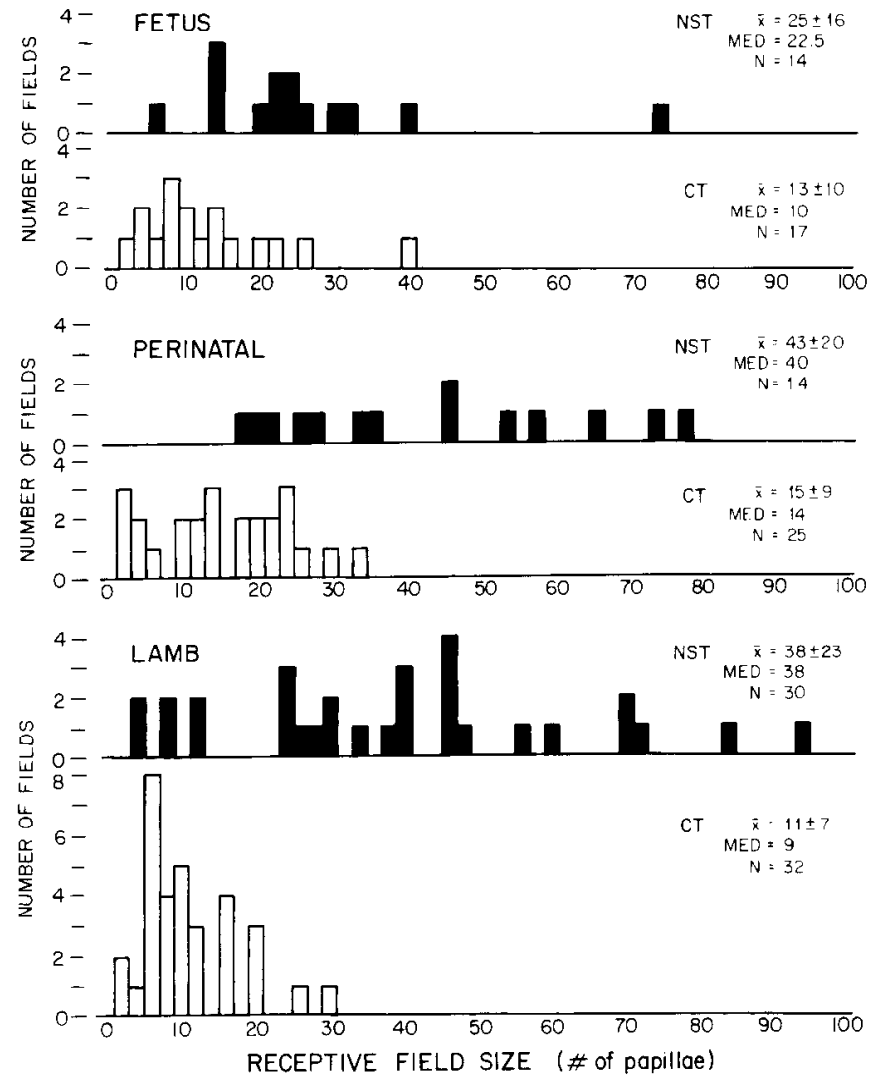

Figure 4. Distributions of numbers of receptive fields of a given size for NST neurons and chorda tympani $(C T)$ fibers in each age group. For each group and neural level, data are provided on mean and SD of field size, median field size $(M E D)$, and number $(N)$ of fields that were studied. Average NST field sizes were significantly larger than CT fields in perinatal animals and lambs, and covered a much wider range. (Data on CT fields from Nagai et al., 1988.)

considerable overlap in receptive fields; that is, receptive fields of separate NST neurons shared many of the same papillae.

Average field sizes for fetal, perinatal, and lamb groups were 25,43 , and 38 papillae, respectively (Fig. 4). This is a significant difference $[F(2,55)=2.63, p=0.08]$ and posttests indicate that receptive fields in fetuses contained fewer papillae on average than fields in perinatal animals or lambs $(p<0.10)$, which did not differ from each other. The difference in average fetal field size was attributable to the virtual absence of very large receptive fields in fetuses (Fig. 4). One fetal field contained 74 papillae but all others had 39 or fewer papillae. This compares with an approximate median field size of about 40 papillae in each of the 2 older age groups. Thus, the receptive field size of NST taste neurons, defined by fungiform papilla input from the tongue, increases between fetal and perinatal ages.

\section{Comparisons between chorda tympani and NST receptive field} size

Comparison of data on receptive field sizes for NST neurons with those from previous studies of chorda tympani fibers (Nagai et al., 1988) provides insight into the basis of the age-related difference for NST fields, and into the extent of convergence from first- to second-order neural levels in the taste system. The average receptive field sizes for chorda tympani fibers in fetal, perinatal, and lamb groups were 13,15 , and 11 papillae, respectively (Fig. 4). In comparison, NST fields were larger (25, 


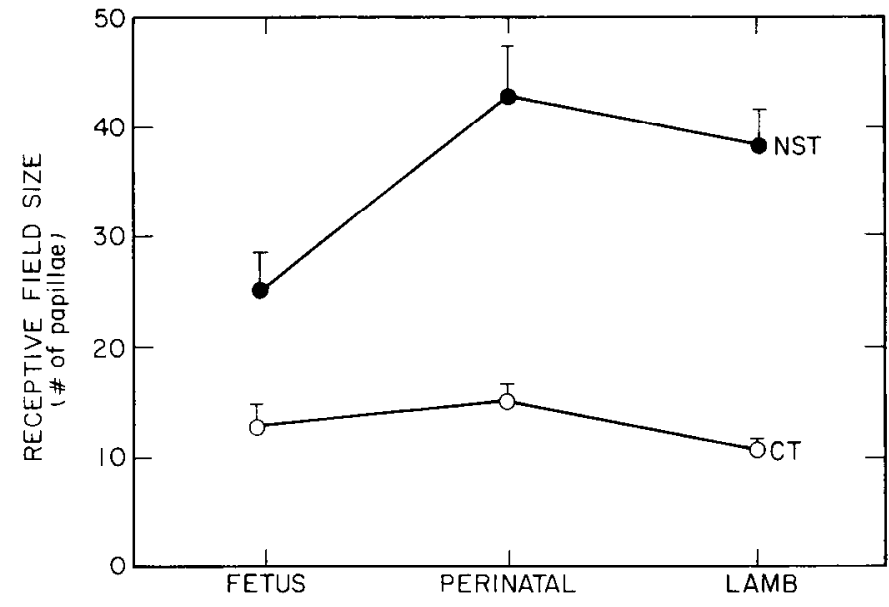

Figure 5. Receptive field sizes for NST neurons and chorda tympani (CT) fibers in fetal, perinatal, and lamb age groups. Data points are means with standard error bars. Not only are NST receptive fields larger than CT fields, but NST fields significantly increase in size during development, whereas CT fields decrease in size (Nagai et al., 1988). This indicates that there is convergence of afferent input onto second-order cells and that the extent of convergence increases developmentally.

43, and 38 papillae) and were distributed over a wider range. Overall, the larger NST receptive fields, relative to chorda tympani fields, indicate that there is convergence of taste papillae input onto second-order neurons.

In addition, there was a marked increase in the extent of convergence between fetal and perinatal ages (Fig. 5). NST receptive field size increased between fetal and perinatal ages, and the increase was sustained in lambs. In contrast, chorda tympani fiber receptive fields decreased in size during development ( $\mathrm{Na}$ gai et al., 1988). Therefore, the changes in NST receptive field size are not simply a reflection of differences in numbers of papillae innervated by chorda tympani fibers, but rather refiect an increase in taste papilla input at second-order neural levels.

Statistical analyses confirmed these observed differences in receptive field sizes of chorda tympani and NST neurons. Twoway analysis of variance including factors of neural level (chorda tympani, NST) and age (fetal, perinatal, lamb) indicated significant effects of neural level $[F(1,126)=79.4, p<0.001]$, age $[F(2,126)=2.79, p=0.06]$, and neural level by age interaction $[F(2,126)=2.82, p=0.06]$. Bonferroni $t$-tests demonstrated that NST receptive fields were larger than chorda tympani fields for perinatal and lamb ages $(p<0.10)$ but not for fetuses.

\section{Relation between salt responses and receptive field size}

We examined the relation between receptive field size and response frequency for NST neurons in each age group (Fig. 6). In lambs, larger receptive fields tended to have higher response frequencies to $\mathrm{NH}_{4} \mathrm{Cl}, \mathrm{NaCl}$, and $\mathrm{KCl}$, and higher spontaneous activity $[r(28)=0.43,0.66,0.39$, and 0.43 , respectively; $p<$ $0.04]$. However, the correlations did not approach significance for neurons in fetal or perinatal animals $(p>0.24)$. The positive correlation between receptive field size and neural responses for lamb NST neurons is consistent with the conclusion that larger receptive fields support greater receptor input and therefore, higher response frequencies. NST neurons in fetal and perinatal animals might not be sufficiently mature to sustain the higher response frequencies generated by input from the larger receptive fields.

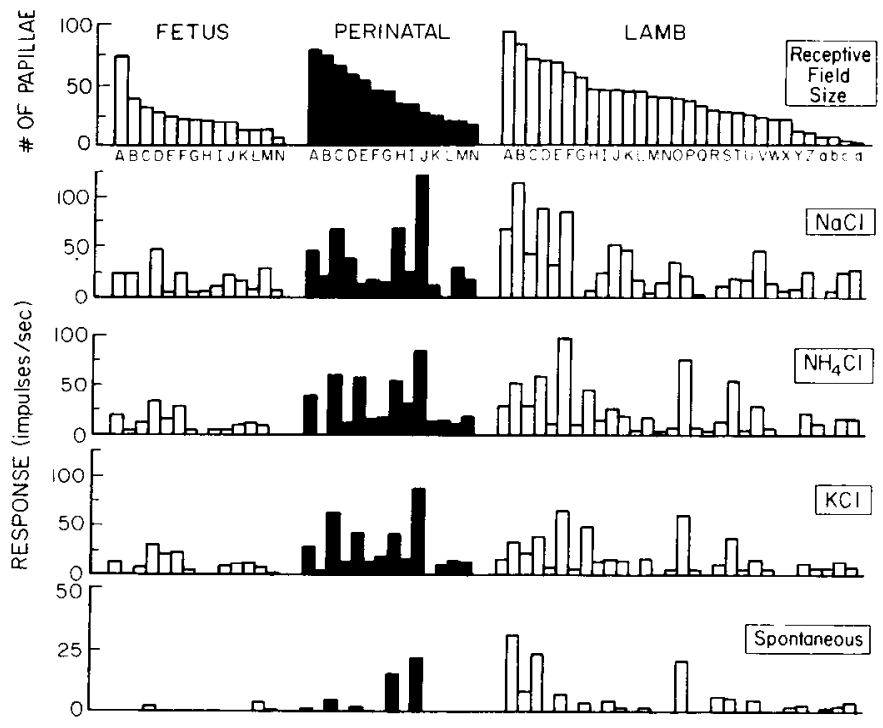

Figure 6. Spontaneous and chemical response frequencies of single NST neurons in each age group compared with receptive field size. Neurons in each group are labeled by letters in the top of the figure, and data for a neuron can be obtained by reading down the columns of response frequencies. In lambs, there was a positive correlation between receptive field size and spontaneous and chemical response frequencies.

For chorda tympani nerve fibers, we had observed a negative correlation between receptive field size and the $\mathrm{NaCl} / \mathrm{NH}_{4} \mathrm{Cl}$ response ratio, defined as the fiber's response frequency to $\mathrm{NaCl}$ divided by the response frequency to $\mathrm{NH}_{4} \mathrm{Cl}$ (Nagai et al., 1988). That is, fibers most responsive to $\mathrm{NaCl}$ had smaller receptive fields than fibers most responsive to $\mathrm{NH}_{4} \mathrm{Cl}$. This relation was observed also for response ratios of NST neurons and receptive field size, by fitting a linear model to data on response ratio versus receptive field size $[r(56)=-0.28, p=0.03$; Fig. 7]. However, 3 small fields with very high response ratios $(33,33$, and 40) influence the correlation; if these fields are removed, the relation is not significant $[r(53)=0.10, p>0.10]$. Further, there is no significant difference in receptive field size for all Na-best versus all NH-best neurons $[t(56)=0.96, p>0.10]$. Therefore, the relation between field size and sodium response sensitivity observed for chorda fibers is lost in NST neurons, presumably because of the convergence of afferent fibers onto second-order cells.

\section{Salt response frequencies across age groups}

It is apparent in Figure 6 that there was considerable variability in chemical response and spontaneous activity rates of NST neurons. However, several trends were evident. Figure 8 presents average chemical and spontaneous impulse frequencies for NST neurons. Included also are data on maximum electrical response frequency, based on the maximum response elicited from any papilla in each receptive field.

Although there is a definite trend for all chemical responses to increase across age groups, only $\mathrm{NH}_{4} \mathrm{Cl}$ and $\mathrm{KCl}$ response frequencies increased significantly $\left[\mathrm{NH}_{4} \mathrm{Cl}: F(2,55)=2.57, p\right.$ $=0.085 ; \mathrm{NaCl}: F(2,55)=1.77, p=0.18 ; \mathrm{KCl}: F(2,55)=2.82$, $p=0.067]$. Posttests demonstrated that the increase for $\mathrm{NH}_{4} \mathrm{Cl}$ and $\mathrm{KCl}$ responses took place between fetal and perinatal stages $(p<0.10)$. Although there appears to be a decrease in chemical responsc frequency between perinatal and lamb groups, the data were not significantly different. Spontaneous activity and max- 


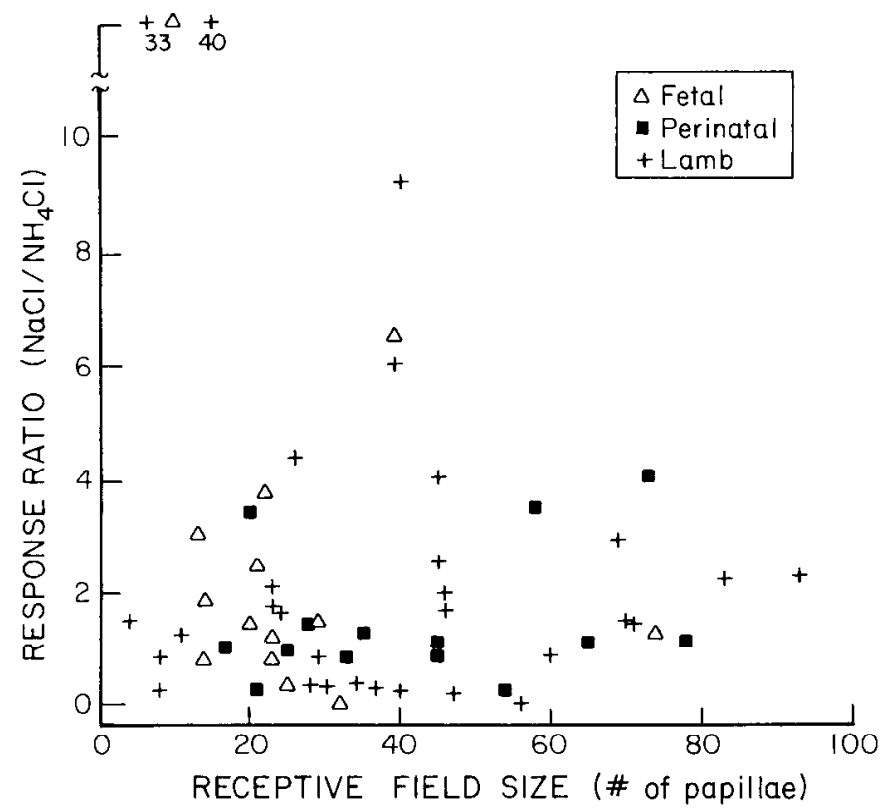

Figure 7. The $\mathrm{NaCl} / \mathrm{NH}_{4} \mathrm{Cl}$ response ratio for each neuron as a function of receptive field size. Data for each group are represented with different symbols. The salt response ratio for all neurons correlates in a negative linear function with receptive field size. However, the correlation is not significant if data on 3 neurons with very high ratios $(33,33,40)$ are excluded from the analysis.

imum electrical response rates did not alter significantly across age groups [spontaneous: $F(2,55)=1.56, p=0.22$; clcctrical: $F(2,55)=1.53, p=0.22$, although an apparent increase for both measures is evident in Figure 8.

Examination of the NST chemical response data by category of neuron, responding with higher frequency to $\mathrm{NH}_{4} \mathrm{Cl}(\mathrm{NH}-$ best) or $\mathrm{NaCl}$ (Na-best), provides insight into the basis for the shapes of the chemical response functions (Fig. 9). Although age-related differences are not statistically significant when neurons are categorized in this way, the data suggest that response frequencies of $\mathrm{NH}$-best neurons alter monotonically during development, whereas frequencies of $\mathrm{Na}$-best cells tend to increase and then decrease. Furthermore, for $\mathrm{NaCl}$ responses only, response frequencies from all $\mathrm{Na}$-best neurons are significantly higher than those from NH-best neurons $[F(1,56)=9.67, p=$ 0.003 ] and this is apparently especially pronounced in the perinatal age group.

\section{Comparisons between chorda tympani and NST response frequencies}

Comparison of NST chemical response frequencies with those from chorda tympani fibers indicates that there are differences in response rates across neural levels for $\mathrm{NaCl}$ and $\mathrm{KCl}$ [Fig. 8; 2-way analysis of variance for neural level versus age group: neural level, $\mathrm{NH}_{4} \mathrm{Cl} F(1,126)=0.29, p>0.10 ; \mathrm{NaCl} F(1,126)$ $=22.8, p<0.0001 ; \mathrm{KCl} F(1,93)=24.4, p=0.001]$. For $\mathrm{NaCl}$ responses, NST frequencies were greater than chorda frequencies at perinatal and lamb ages (Bonferroni $t$-tests, $p<0.10$ ). For $\mathrm{KCl}$ responses, NST frequencies were greater than chorda frequencies in the lamb group $(p<0.10)$.

Spontaneous rates also differed across neural levels $[F(1,126)$ $=7.02, p=0.009$ ], and were significantly lower in NST neurons than in chorda fibers in the fetal age group $(p<0.10)$. Differences
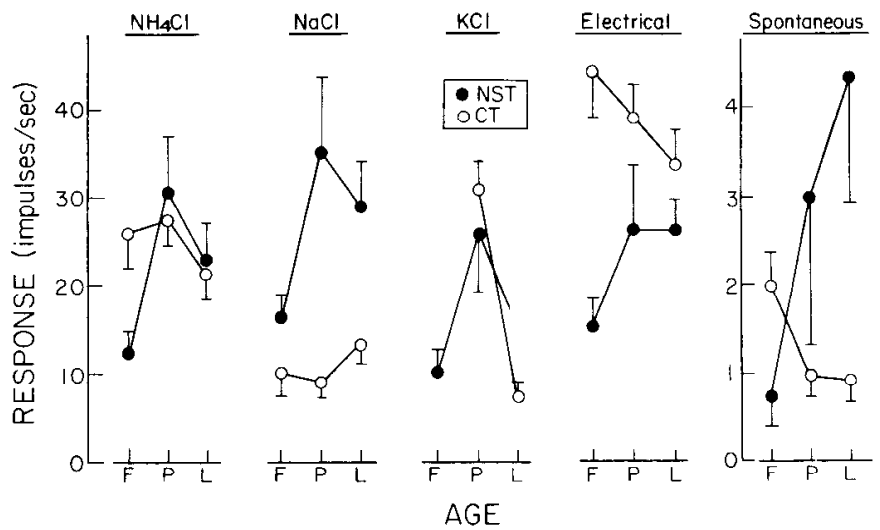

Figure 8. Response frequencies for NST neurons and chorda tympan. (CT) fibers from fetal, perinatal, and lamb age groups $(F, P, L)$ during chemical and electrical stimulation of papillae, and spontaneous activity. Data are presented as means and standard error bars for each age group. NST responses to $\mathrm{NH}_{4} \mathrm{Cl}$ and $\mathrm{KCl}$ increased significantly between fetal and perinatal groups; other apparent differences for $\mathrm{NaCl}$, electrical, and spontaneous activity were not significant. Responses to $\mathrm{NaCl}, \mathrm{KCl}$, electrical stimulation, and spontaneous activity differed significantly across ncural levels.

were also observed for the maximum electrical response $[F(1$, $126)=20.2, p<0.0001]$. As with spontaneous frequencies, the maximum electrical response was lower in NST neurons than in chorda fibers in the fetal age group $(p<0.10)$.

In addition to examination of average response frequencies, it is informative to categorize neurons on the basis of salt responses (Table 1). Although most NST neurons responded to all salt stimuli, relative sensitivity differed: $66 \%$ of total neurons responded with highest frequency to $\mathrm{NaCl}$ and $34 \%$ to $\mathrm{NH}_{4} \mathrm{Cl}$. This contrasts sharply with data for chorda tympani nerve fibers (Nagai et al., 1988). Of total chorda fibers, only $27 \%$ responded with highest frequency to $\mathrm{NaCl}$ (Table 1), whereas $73 \%$ responded to $\mathrm{NH}_{4} \mathrm{Cl}$. Separate chi-square tests for NST neurons responding best to $\mathrm{NaCl}$ and $\mathrm{NH}_{4} \mathrm{Cl}$ indicated that the proportion of 1 .eurons in each category did not differ across age groups $\left(\chi^{2}\right.$, $2 \mathrm{df}$, $=0.49$ and $1.36, p>0.10$ ). Again, this contrasts with results for chorda tympani fibers, because the proportion of fibers responding best to $\mathrm{NaCl}$ increased across age groups $(\mathrm{Na}-$ gai et al., 1988).

Therefore, NST neurons differed from chorda tympani fibers in salt response, spontaneous and electrical frequencies, and in the greater proportion of neurons more responsive to $\mathrm{NaCl}$ than to $\mathrm{NH}_{4} \mathrm{Cl}$.

\section{Chemical response adaptation during development}

To determine adaptation characteristics for salt responses of NST neurons across age groups, we calculated the linear slope of the response frequency during seconds 2-7 of the response period. The frequency for the first second of the response was not included because we were interested in the steady state portion of the response. In addition, the initial 1 sec of the response was usually very high relative to the later portion of the response, thus contributing to slope values that were artificially steep.

Before calculating response slopes, we identified those responses that adapted completely; that is, those with a neural discharge that adapted to spontaneous frequency rates before the salt stimulus was rinsed from the tongue. In general, the proportion of responses that adapted completely was greater in 


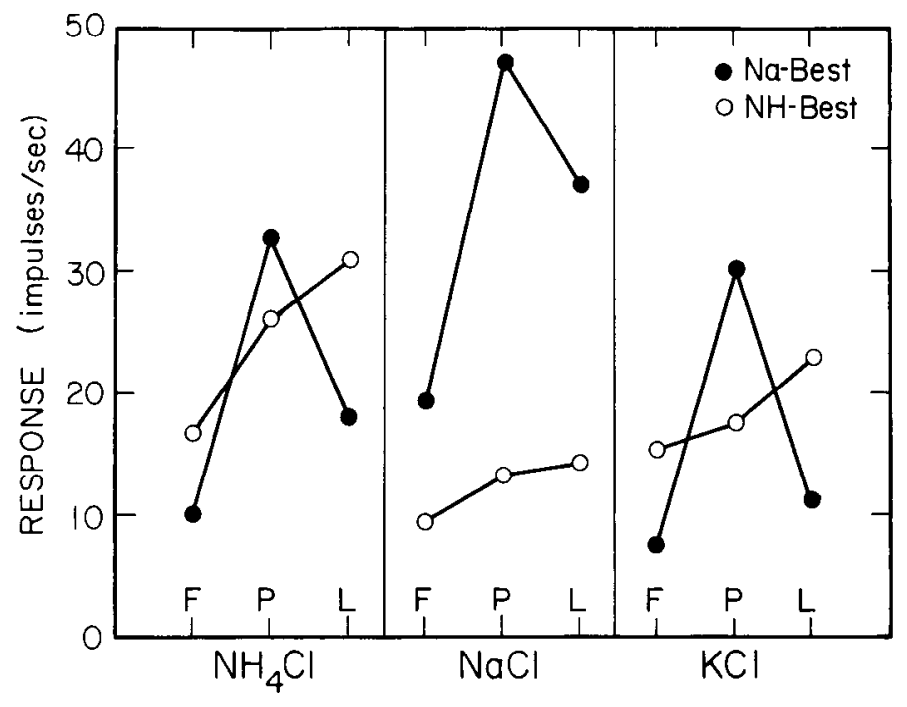

Figure 9. Average salt response frequencies for NST neurons categorized as $\mathrm{Na}$-best (response frequency to $\mathrm{NaCl}$ higher than to $\mathrm{NH}_{4} \mathrm{Cl}$ ) or $\mathrm{NH}$-best (response frequency to $\mathrm{NH}_{4} \mathrm{Cl}$ higher than to $\mathrm{NaCl}$ ). Response frequencies for fetal, perinatal, and lamb age groups $(F, P, L)$ are indicated. Functions for NH-best neurons tend to alter monotonically with age, whereas functions for Na-best cells tend to increase and then decrease.

fetal $(28 \%)$ and perinatal $(26 \%)$ animals than in lambs $(10 \%)$. For chemicals, $25 \%$ of $\mathrm{KCl}$ responses adapted completely, $16 \%$ of $\mathrm{NH}_{4} \mathrm{Cl}$, and $17 \%$ of $\mathrm{NaCl}$. These differences were not significantly different with $\chi^{2}$ analysis.

Response slopes for neurons that did not adapt completely were calculated (Fig. 10). For each salt, slopes differed significantly across age groups $\left[\mathrm{NH}_{4} \mathrm{Cl}: F(2,43)=5.16, p=0.01\right.$; $\mathrm{NaCl}: F(2,42)=10.03, p=0.0003$; KCl: $F(2,35)=6.73, p=$ $0.003]$. However, the functions were not monotonic with age. Slopes were steepest (greatest degree of response adaptation) in the perinatal age group. Bonferroni $t$-tests indicated that slopes for perinatal responses to $\mathrm{NaCl}$ were more negative than those of fetuses or lambs; slopes for perinatal responses to $\mathrm{NH}_{4} \mathrm{Cl}$ and $\mathrm{KCl}$ were more negative than those of lambs $(p<0.05$, based on 3 post hoc comparisons per chemical).

Although not as steep as perinatal slopes, fetal neurons also had substantial adaptation slopes for $\mathrm{NaCl}$ and $\mathrm{KCl}$ responses. In contrast, neurons in lambs had slopes near zero, demonstrating little adaptation, or had positive slopes, demonstrating some tendency for response frequencies to be maintained or actually increase during the stimulus period.

\section{Discussion}

\section{Neuroanatomical basis of convergence}

Comparison of receptive field sizes of NST neurons and chorda tympani nerve fibers demonstrates that there is marked convergence of taste receptor input onto second-order taste cells. In perinatal animals and lambs, receptive fields of NST neurons average about 40 fungiform papillae compared with about 12 papillae in chorda tympani fiber fields. Furthermore, comparison of receptive field size in animals of different ages reveals developmental changes in the extent of afferent convergence. Receptive fields of NST neurons increase nearly 2 -fold in size between fetal and perinatal ages. On the other hand, receptive field size of chorda tympani fibers does not increase during this period. Thus, because the increase in NST field size cannot be
Table 1. Percentage of Na-best (responding with highest frequency to $\mathrm{NaCl}$ ) and $\mathrm{NH}$-best (responding with highest frequency to $\mathrm{NH}_{4} \mathrm{Cl}$ ) neurons in the chorda tympani nerve and NST in 3 age groups of sheep

\begin{tabular}{|c|c|c|c|c|}
\hline & \multicolumn{4}{|c|}{ Na-best neurons (\%) } \\
\hline & Fetal & Perinatal & Lamb & All \\
\hline Chorda tympani & 12 & 20 & 41 & 27 \\
\hline \multirow[t]{2}{*}{ NST } & 71 & 64 & 63 & 66 \\
\hline & \multicolumn{4}{|c|}{ NH-best neurons (\%) } \\
\hline Chorda tympani & 88 & 80 & 59 & 73 \\
\hline NST & 29 & 36 & 37 & 34 \\
\hline
\end{tabular}

From fetal through lamb stages, the proportion of Na-best neurons in the NST was high compared with the proportion in the chorda tympani nerve.

attributed to an increase in the number of papillae innervated by chorda tympani fibers, the expansion in second-order fields most likely reflects an increase in afferent fiber input to NST neurons.

An alternative cxplanation to afferent convergence for the larger receptive field sizes in second-order neurons could be a network of excitatory collaterals between NST neurons, or excitatory connections via interneurons. To account for the increase in receptive fields of second-order cells during development, the excitatory interconnections would have to increase also. Unfortunately there is scant direct evidence related to this issue for the solitary nucleus al any age or in any species. Evidence that does exist suggests the presence of a small proportion of inhibitory interneurons only, based on synaptic characteristics (Whitehead, 1986) and morphology of neurons in Golgi preparations from adult hamster (Whitehead, 1988), and on immunocytochemistry in adult rat (Lasiter and Kachele, 1988) and hamster (Davis and Jang, 1988).

NST neurons from sheep have complex morphologies that could support converging afferent input. Preliminary data (Gurkan and Mistretta, 1989) and work in progress in our laboratory describe the morphology of cells in regions of sheep rostral gustatory NST that exhibit high-frequency responses to anterior tongue stimulation with salts. All of the cells have multiple dendrites and some have highly complex dendritic arrangements from at least $110 \mathrm{~d}$ of gestation through postnatal lamb stages. In the peripheral autonomic nervous system there is evidence that the number of dendrites on a postsynaptic cell correlates with the number of innervating axons (Purves and Hume, 1981). Thus, there is more convergent input on cells with a more complex geometry. Our preliminary work indicates that neuronal geometry in rostral gustatory NST of sheep is compatible with extensive convergent input from taste fibers. We do not yet know how neuronal geometry alters in sheep during the developmental period of functional convergence. In postnatal rat NST there is an increase in dendritic length of rostral neurons during periods when response characteristics of the cells are altering (Lasiter et al., 1989). The greater dendritic extent could support converging input.

Convergence of afferent input onto second-order cells has been observed also in postnatal development of rat dorsal horn cells (Fitzgerald, 1985). Over the first 2 postnatal weeks, low and high threshold cutaneous inputs converge in increasing proportion onto lumbar dorsal horn neurons. During the period of afferent convergence, receptive field size reportedly decreases; 


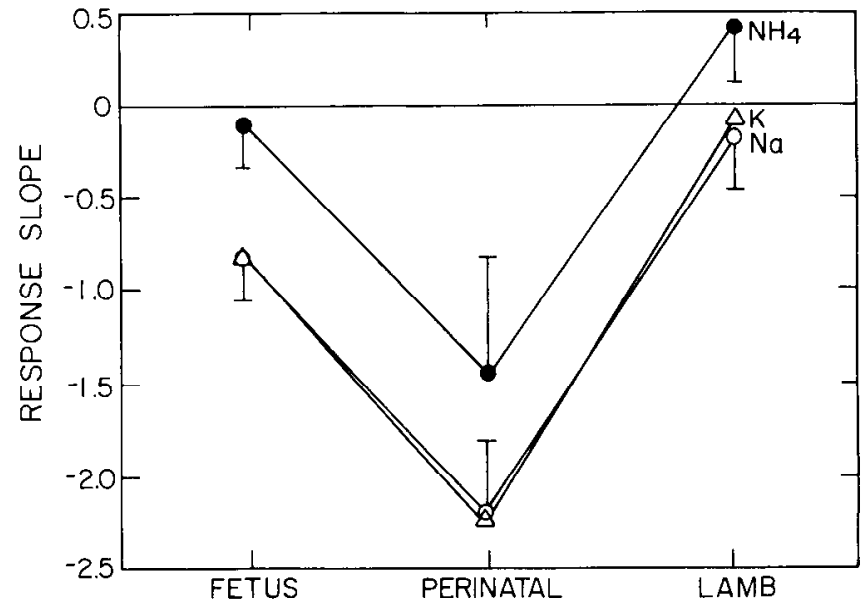

Figure 10. Response slopes (linear slope of response frequency vs time) as a measure of neural adaptation during stimulation of the tongue with $\mathrm{NH}_{4} \mathrm{Cl}, \mathrm{KCl}$, and $\mathrm{NaCl}$ for NST neurons in fetal, perinatal, and lamb groups. Data points are means with standard error bars. For each salt, adaptation characteristics, as measured by response slopes, differed significantly across age groups.

however, the receptive field measure was area of skin in proportion to total hindlimb area, not a quantification of receptor organ number. The decrease in receptive field area was attributed to a proposed postnatal increase in inhibitory interneurons in the dorsal horn. In cuneate nucleus of postnatal kitten investigators have reported an absence of developmental convergence from afferent tactile fibers (Ferrington and Rowe, 1982). At birth the highly specific, adult pattern of afferent input onto 3 classes of cuneate neurons is already established. Convergent input can actually be decreased, also, as in development of the chick auditory system. Soon after hatching, an average of 4 axons of the eighth nerve contact neurons in the cochlear nucleus; this number is reduced over 2 weeks to about 2 axons per neuron (Jackson and Parks, 1982). Other systems, therefore, have varying developmental sequences to establish the adult pattern of afferent connections onto second-order neurons.

\section{Neural activity and receptive feld size}

The increase in receptive field size of NST neurons between fetal and perinatal age groups is accompanied by an increase in neural response frequencies to salts. Also, relative to chorda tympani fibers, spontaneous activity and electrical response frequencies are increased. This increase in neural frequencies is another indicator of increasing convergence of peripheral input onto second-order cells. In studies of NST responses during development in rat, investigators also reported increases in salt response frequencies and spontaneous activity, and speculated that convergent afferent input could underlie the change (Hill et al., 1983).

The high salt response frequencics observed in perinatal NST neurons in sheep are apparently too high to be maintained by the still immature, second-order cells. Thus, the response discharge of neurons at this age adapts more rapidly to salt stimuli than in fetal or postnatal cells (Fig. 10). This is a characteristic of immature neurons in general and has been reported previously for fetal taste neurons (Bradley and Mistretta, 1980). Immature adaptation characteristics may relate to increasing convergence of afferent input at a time when the second-order cells are not yet sufficiently mature to sustain high frequency discharges.

It is notable that whereas frequencies increase in NST neurons between fetal and perinatal stages, chorda tympani fiber frequencies either remain the same $\left(\mathrm{NH}_{4} \mathrm{Cl}, \mathrm{NaCl}\right.$ responses) or actually decrease (electrical responses, spontaneous activity) during this period (Fig. 8). Indeed the establishment of extensive converging afferent input between fetal and perinatal stages might only be possible because peripheral input frequencies to NST neurons are low. Thus, competition among afferent fibers for second-order targets could be minimized and permit multiple innervation of single neurons (as discussed in Purves and Lichtman, 1985).

It is important that although the quantity of salt taste input to the NST remains low during the period of increase in NST receptive field size (from fetal to perinatal stages), the pattern of salt taste activity transmitted by peripheral nerves is altering. During this time and continuing postnatally, there is an increase in the proportion of chorda tympani fibers that respond with highest frequency to anterior tongue stimulation with $\mathrm{NaCl}$, compared to fibers that respond with highest frequency to $\mathrm{NH}_{4} \mathrm{Cl}$ (Nagai et al., 1988). The proportion of NaCl-best chorda tympani fibers increases from 12 to $41 \%$ from fetal to postnatal stages (Table 1). Therefore, the nature of the peripheral input related to salt taste sensation alters substantially during this time and might influence the type of second-order connections that are made. Because fetuses swallow amniotic fluid (Wintour, 1986), and other secretions also bathe the taste buds, a natural and changing source of taste stimulation is available in utero (Mistretta and Bradley, 1975). Of course, there is also opportunity for spontaneous activity to have a role in organization of salt taste circuits, as demonstrated during fetal development of the mammalian visual system (Galli and Maffei, 1988; Shatz and Stryker, 1988).

\section{Role of convergence in the NST}

Convergence is a general characteristic of sensory pathways that scrves to intcgrate afferent input for central processing. The establishment of convergent input to gustatory NST in prenatal periods might function to maximize gain in salt taste systems so that the relatively weak peripheral nerve taste responses to $\mathrm{NaCl}$ can be processed centrally. Gain can be maximized either by intrinsic properties at synapses or by convergence of many synapses onto 1 neuron (Koch et al., 1986). A low presynaptic input can be amplified by increasing the number of synapses transmitting information to the postsynaptic cell, if synaptic saturation is avoided. Distribution of peripheral input at relatively distant sites on second-order dendrites can minimize such saturation, so postsynaptic neuronal geometry is important.

Our data suggest that the development of convergence in sheep NST functions to maximize gain during differentiation of $\mathrm{NaCl}$ sensory circuits. The very low neural response frequency to $\mathrm{NaCl}$ in chorda tympani fibers is amplified more than 3 times in NST neurons (Fig. 8). Furthermore, in contrast to the increasing proportion of Na-best chorda tympani fibers during development, the proportion of Na-best neurons in the NST is high initially and remains high. From fetal to perinatal to postnatal stages, Na-best neurons are 71,64 , and $63 \%$, respectively, of the total population (Table 1). This suggests that convergent afferent fiber input bestows the property of high sodium responsiveness on second-order neurons, even at fetal ages when peripheral responses to $\mathrm{NaCl}$ are very weak relative to $\mathrm{NH}_{4} \mathrm{Cl}$ 
or $\mathrm{KCl}$. Interestingly, the proportion of $\mathrm{NH}$-best neurons remains relatively low in the NST across age groups. Therefore, the nature of the convergence that takes place apparently maximizes $\mathrm{NaCl}$ input in particular.

The extensive convergence of sodium input eradicates the relation between receptive field size and $\mathrm{NaCl}$ responsiveness that was observed in the peripheral taste system. In the periphery, receptive fields more highly responsive to $\mathrm{NaCl}$ are smaller than those more responsive to $\mathrm{NH}_{4} \mathrm{Cl}$ (Nagai et al., 1988). This relation apparently does not hold in NST neurons, presumably because afferent convergence maximizes $\mathrm{NaCl}$ responsiveness in the NST.

Although the evidence for convergence has not been direct, others have attributed the increased range of chemical responsiveness and higher response frequencies of NST neurons, compared to chorda tympani fibers, to convergence (Doetsch and Erickson, 1970; Travers and Smith, 1979; Hill et al., 1983). Extensive convergence in NST might stabilize central neural responses during the continuing process of receptor cell turn over and replacement in the taste system (Beidler and Smallman, 1965). However, convergence in the periphery of numerous taste cells onto single terminal branches and of multiple taste buds onto single chorda tympani fibers is probably effective in stabilization.

A primary contribution of our study of receptive fields of NST neurons is the quantitative assessment of field sizes of mammalian central neurons during development. Such quantitative measures are difficult in most other sensory systems but are possible in taste because individual fungiform papillae are discretely located and the taste buds within these papillae respond to electrical stimulation (Herness, 1985). Other investigators have demonstrated a more general type of convergence from taste receptors in widely different parts of the oral cavity, for example nasoincisor duct and anterior tongue, or soft palate and posterior tongue, onto single NST neurons (Travers et al., 1986; Sweazey and Bradley, 1989). However, to our knowledge there are no other published data on receptive field size of central taste neurons that actually quantify receptor units.

\section{Order preserved during convergence}

Even in view of the extensive convergence from first- to secondorder cells in the taste system, a high degree of order is preserved. For example, the receptive fields of NST neurons are composed of fungiform papillae that are clustered together; nonresponsive papillae are not interspersed among responsive ones, nor are "split" fields observed with a cluster of papillae in 1 location and another cluster in a very separate tongue location. This same property of receptive field coherence was observed for chorda tympani fibers (Nagai et al., 1988). Therefore, the large NST fields must receive input from chorda tympani fibers with smaller, but contiguous or overlapping, receptive fields. The developmental mechanism by which this order is maintained among chorda tympani projections is unknown. It might result from neural proximity, so that axons innervating contiguous receptive fields project centrally in discrete bundles and converge on central neurons. In the trigeminal system of developing rodents, there is evidence for (Erzurumlu and Killackey, 1982) and against (Davies and Lumsden, 1986) a role for ordered fascicles of nerve fibers in maintaining the characteristic topographic order in brain-stem projections from whisker follicle afferents.

Because we sometimes observed overlap in the receptive fields of individual NST neurons recorded in 1 animal, we know that there also is divergence of some afferent fibers to contact more than 1 second-order neuron. However, we did not systematically study the question of overlapping fields for multiple neurons in single animals, and so have no quantitative estimate of the extent of divergence.

\section{Summary and Conclusions}

During functional differentiation of sensory pathways for salt taste, first-order taste afferents converge on second-order neurons and the extent of convergence increases from fetal through postnatal stages in sheep. Several measures lead to the conclusion of convergence. Receptive field sizes of NST neurons increase during development, whereas chorda tympani fiber fields decrease over the same stages. Spontaneous activity and response frequencies for some salts increase during development in NST, whereas comparable chorda tympani fiber activity either decreases or remains the same.

One possible role of this increasing convergence is to amplify weak peripheral input about $\mathrm{NaCl}$ taste stimuli to central neural relays. This amplification is manifest in the higher NST response frequencies to $\mathrm{NaCl}$ in perinatal animals and lambs, compared with chorda tympani frequencies at these ages. In contrast, NST response frequencies for $\mathrm{NH}_{4} \mathrm{Cl}$ are not higher than chorda frequencies. Also, the proportion of $\mathrm{Na}$-best neurons is very high in NST relative to the chorda tympani, even in fetal stages.

Altering salt taste responses in the chorda tympani nerve provides a changing neural input pattern to the NST during development and may play a role in effecting convergence. In addition to altering neural input, the rather complex geometry of NST neurons, manifest to some degree even in fetuses, might play a permissive role in establishing convergence in NST.

The quantification of taste receptive field sizes at 2 neural levels provides strong evidence for an increase in convergence in the NST over the 3 age groups studied. The increasing afferent input apparently functions to increase gain for $\mathrm{NaCl}$ taste sensation. Therefore, neural rearrangements during differentiation of salt taste pathways result in specific functional outcomes.

\section{References}

Beidler LM, Smallman RL (1965) Renewal of cells within tastc buds. J Cell Biol 27:263-272.

Bradley RM, Mistretta CM (1973) The gustatory sense in foetal sheep during the last third of gestation. J Physiol 231:271-282.

Bradley RM, Mistretta CM (1980) Developmental changes in neurophysiological taste responses from the medulla in sheep. Brain Res 191:21-34.

Bradley RM, Sweazey RD (1990) In vitro intracellular recordings from gustatory neurons in the rat solitary nucleus. Brain Res 508:168-171.

Davies AM, Lumsden AGS (1986) Fasciculation in the early mouse trigeminal nerve is not ordered in relation to the emerging pattern of whisker follicles. J Comp Neurol 253:13-24.

Davis BJ, Jang T (1988) Tyrosine hydroxylase-like and dopamine B-hydroxylase-like immunoreactivity in the gustatory zone of the nuclcus of the solitary tract in the hamster: light- and electron-microscopic studies. Neuroscience 27:949-964.

Doetsch GS, Erickson RP (1970) Synaptic processing of taste quality information in the nucleus tractus solitarius of the rat. J Neurophysiol 33:490-507.

Donoghue S, Felder RB, Gilbey MP, Jordan D, Spyer KM (1985) Postsynaptic activity evoked in the nucleus tractus solitarius by carotid sinus and aortic nerve afferents in the cat. J Physiol 360:261-273. 
Erzurumlu RS, and Killackey HP (1982) Order in the developing rat trigeminal nerve. Dev Brain Res 3:305-310.

Ferrington DG, Rowe MJ (1982) Specificity of connections and tactile coding capacities of cuneate nucleus of the neonatal kitten. J Neurophysiol 47:622-640.

Fitzgerald M (1985) The post-natal development of cutaneous afferent fibre input and receptive field organization in the rat dorsal horn. J Physiol 364:1-18.

Frank ME, Hettinger TP, Herness MS, Pfaffmann C (1986) Evaluation of taste function by electrogustometry. In: Clinical measurement of taste and smell (Meiselman HL, Rivlin RS, eds), pp 187-199. New York: Macmillan.

Galli L, Maffei L (1988) Spontaneous impulse activity of rat retinal ganglion cells in prenatal life. Science 242:90-91.

Gurkan S, Mistretta CM (1989) Morphology of neurons in the anterior tongue projection of the nucleus of the solitary tract during development in sheep. Chemical Senses 14:706.

Herness MS (1985) Neurophysiological and biophysical evidence on the mechanism of electrical taste. J Gen Physiol 86:59-87.

Hill DL, Bradley RM, Mistretta CM (1983) Development of taste responses in rat nucleus of solitary tract. J Neurophysiol 50:879-895.

Jackson H, Parks TN (1982) Functional synapse elimination in the developing avian cochlear nucleus with simultaneous reduction in cochlear nerve axon branching. J Neurosci 12:1736-1743.

Jacquin MF, Barcia M, Rhoades RW (1989) Structure-function relationships in rat brainstem subnucleus interpolaris: IV. Projection neurons. J Comp Neurol 282:45-62.

Kitada Y, Bradley RM, Mistretta CM (1984) Maintenance of chorda tympani salt taste responses after nerve transection in rats. Brain Res 302:163-170.

Koch C, Poggio T, Torre V (1986) Computations in the vertebrate retina: gain enhancement, differentiation and motion discrimination. Trends Neurosci 9:204-211.

Lasiter PS, Kachele DL (1988) Organization of GABA- and GAGAtransaminasc containing ncurons in the gustatory zone of the nucleus of the solitary tract. Brain Res Bull 21:623-636.

Lasiter PS, Wong DM, Kachele DL (1989) Postnatal development of the rostral solitary nucleus in rat: dendritic morphology and miluchondrial enzyme activity. Brain Res Bull 22:313-321.

Lawson EE, Richter DW, Ballantyne D, Lalley PM (1989) Peripheral chemoreceptor inputs to medullary inspiratory and postinspiratory neurons of cats. Pflugers Arch 414:523-533.

Madden KP, Remmers JE (1982) Short time correlations between spike activity of neighboring respiratory neurons of nucleus tractus solitarius. J Neurophysiol 48:749-760.
Mifflin SW, Felder RB (1988) An intracellular study of time-dependent cardiovascular afferent interactions in the nucleus tractus solitarius. J Neurophysiol 59:1798-1813.

Mistretta CM, Bradley RM 1975) Taste and swallowing in utero: a discussion of fetal sensory function. Brit Med Bull 31:80-84.

Mistretta CM, Bradley RM (1983) Neural basis of developing salt taste sensation: Response changes in fetal, postnatal and adult sheep. J Comp Neurol 215:199-210.

Mistretta CM, Gurkan S, Bradley RM (1988) Morphology of chorda tympani fiber receptive fields and proposed neural rearrangements during development. J Neurosci 8:73-78.

Nagai T, Mistretta CM, Bradley RM (1988) Developmental decrease in size of peripheral receptive fields of single chorda tympani nerve fibers and relation to increasing $\mathrm{NaCl}$ taste sensitivity. J Neurosci 8: 64-72.

Purves D, Hume RI (1981) The relation of postsynaptic geometry to the number of presynaptic axons that innervate autonomic ganglion cells. J Neurosci 1:441-452.

Purves D, Lichtman JW (1985) Principles of neural development. Sunderland, MA: Sinauer.

Scott TR, Yaxley S, Sienkiewicz ZJ, Rolls ET (1986) Gustatory responses in the nucleus tractus solitarius of the alert cynomolgus monkey. J Neurophysiol 55:182-200.

Shatz CJ, Stryker MP (1988) Prenatal tetrodotoxin infusion blocks segregation of retinogeniculate afferents. Science 242:87-89.

Shepherd GM (1983) Neurobiology. Oxford: Oxford University Press.

Smith DV, Van Buskirk RL, Travers JB, Bieber SL (1983) Gustatory neuron types in hamster brain stem. J Neurophysiol 50:522-540.

Sweazey RD, Bradley RM (1989) Responses of neurons in the lamb nucleus tractus solitarius to stimulation of the caudal oral cavity and epiglottis with different stimulus modalities. Brain Res 480:133-150.

Travers JB, Smith DV (1979) Gustatory sensitivities in neurons of the hamster nucleus tractus solitarius. Sensory Processes 3:1-26.

Travers S, Pfaffmann C, Norgren R (1986) Convergence of lingual and palatal gustatory ncural activity in the nuclcus of the solitary tract. Brain Res 365:305-320.

Whitehead MC (1986) Anatomy of the gustatory system in the hamster: synaptology of facial afferent terminals in the solitary nucleus. $J$ Comp Neurol 244:72-85.

Whitehead MC (1988) Neuronal architecture of the nucleus of the solitary tract in the hamster. J Comp Neurol 276:547-572.

Wintour EM (1986) Amniotic fluid-our first environment. News Physiol Sci. 1:95-97. 